\title{
SOME ELEMENTARY TOPOLOGICAL PROPERTIES OF ESSENTIAL MAXIMAL MODEL CONTINUA
}

\author{
W. R. SCOTT ${ }^{1}$
}

1. Introduction. Let $T: z=t(w)$, $w \in D$, be a bounded continuous transformation from a bounded connected open set $D$ in the w-plane into the z-plane. Radó and Reichelderfer [3, p. 263 $]^{2}$ have defined essential maximal model continua $\gamma(z)$ for such transformations. If $A$ is a set in the $z$-plane, let $E(A)$ be the point set sum of the e.m.m.c. $\gamma(z)$ for $z \in A$. Thus $E(A)$ is a subset of $D$. We are primarily interested in the topological properties of $E(A)$. Since most of the reasoning is valid for more general spaces, it is carried out first for these spaces and then in $\$ 6$ the results are applied to the above situation. These results are also applicable to the set $E^{+}(A)$ and $E^{-}(A)$ defined by Reichelderfer in [4].

2. Preliminaries. Let $T: y=t(x), x \in X$, be a single-valued continuous transformation from a normal Hausdorff space $X$ into a topological space $Y$ (these terms are used in the sense of $[1$, chap. 1]). Suppose that for each set $A \subset Y$ there has been defined a set $E(A) \subset X$ such that

$$
\begin{aligned}
& E(A)=\sum_{y \in A} E(y), \\
& E(y)=\sum_{\gamma \in S(y)} \gamma,
\end{aligned}
$$

where $S(y)$ is a class of disjoint nonempty closed connected sets $\gamma \subset X$ such that $\gamma \subset T^{-1}(y)$ and having the following property:

P. If $\gamma_{0} \in S\left(y_{0}\right)$ and if $G$ is any open set such that $\gamma_{0} \subset G$, then there exists a neighborhood $N\left(y_{0}\right)$ such that if $y \in N\left(y_{0}\right)$ then there exists a $\gamma \in S(y)$ such that $\gamma \subset G$.

Let $f(y)$ be the number of $\gamma \in S(y)$. Thus $f(y)=0,1,2, \cdots$, or $+\infty$, no distinction being made between infinite cardinals.

\section{Closed sets $A$.}

Theorem 1. Let $T, A, E(A)$, and $f(y)$ be given as in \$2. If $A$ is closed and if $f(y), y \in A$, is upper semi-continuous and bounded on $A$, then $E(A)$ is closed.

Received by the editors May 27, 1948.

1 This paper was written under the joint sponsorship of the U.S. Navy, Office of Naval Research, and the Ohio State University Research Foundation.

${ }^{2}$ Numbers in brackets refer to the bibliography at the end of the paper. 
Proof. Deny the theorem. Then there exists a point $x_{0} \in C(E(A))$ $-E(A)$, where $C(E(A))$ is the closure of $E(A)$. Since $A$ is closed and since $E(A) \subset T^{-1}(A)$ which is closed, we have $t\left(x_{0}\right)=y_{0} \in A$. Let $f\left(y_{0}\right)=k$. Then there exist disjoint $\gamma_{i} \in S\left(y_{0}\right), i=1, \cdots, k$, and $x_{0} \cdot \sum \gamma_{i}=0$. Since each $\gamma_{i}$ is closed, and since $X$ is a normal Hausdorff space, there exist disjoint open sets $G_{0}, \cdots, G_{k}$, such that $x_{0} \in G_{0}$, $\gamma_{i} \subset G_{i}, i=1, \cdots, k$. By property $\mathrm{P}$, there exist neighborhoods $N_{i}\left(y_{0}\right)$ such that if $y \in N_{i}\left(y_{0}\right)$ then there exists a $\gamma \in S(y)$ such that $\gamma \subset G_{i}$. Since $f(y)$ is upper semi-continuous on $A$, there is a neighborhood $N^{\prime}\left(y_{0}\right)$ such that if $y \in N^{\prime}\left(y_{0}\right) \cdot A$, then $f(y) \leqq k$. Let

$$
N\left(y_{0}\right)=N^{\prime}\left(y_{0}\right) \cdot N_{1}\left(y_{0}\right) \cdots N_{k}\left(y_{0}\right) \text {. }
$$

Then if $y \in N\left(y_{0}\right) \cdot A$, all of the $\gamma \in S(y)$ lie in $\sum_{i=1}^{k} G_{i}$. But since $x_{0} \in G_{0}$ and $G_{0} \cdot \sum_{i=1}^{k} G_{i}=0$, this implies that $x_{0} \notin C(E(A))$. This is a contradiction. Thus $E(A)$ is closed.

It will be noted that the fact that each $\gamma$ is connected was not used in the proof of this theorem.

\section{Connected sets $A$.}

LEMMA 1. Let $T, A$, and $E(A)$ be given as in $\$ 2$. Let $E(A)=E_{1}+E_{2}$ where $E_{1} \cdot C\left(E_{2}\right)+C\left(E_{1}\right) \cdot E_{2}=0$. Let $f_{i}(y), y \in A$, be the number of $\gamma \in S(y)$ such that $\gamma \subset E_{i}$. Then $f_{i}(y)$ is lower semi-continuous on $A$.

Proof. Note that since a $\gamma_{0} \in S\left(y_{0}\right)$ is connected, either $\gamma_{0} \subset E_{i}$ or or $\gamma_{0} \cdot E_{i}=0$. Hence $f_{1}(y)+f_{2}(y)=f(y), y \in A$. It will be sufficient to show that $f_{1}(y)$ is lower semi-continuous on $A$. Let $f_{1}\left(y_{0}\right) \geqq n, y_{0} \in A$. If $n=0$, then $f_{1}(y) \geqq n, y \in A$. If $n>0$, there exist $\gamma_{j} \subset E_{1} \cdot E\left(y_{0}\right)$, $j=1, \cdots, n$. Hence $\gamma_{1}, \cdots, \gamma_{n}$, and $C\left(E_{2}\right)$ are disjoint closed sets. Therefore there exist disjoint open sets $G_{1}, \cdots, G_{n}$ such that $C\left(E_{2}\right) \cdot \sum G_{j}=0$ and $\gamma_{j} \subset G_{j}$. By property $\mathrm{P}$ there exists a neighborhood $N\left(y_{0}\right)$ such that if $y \in N\left(y_{0}\right)$ then there exists a $\gamma_{j} \subset G_{j} \cdot E(y)$. If, in addition, $y \in A$, then $f_{1}(y) \geqq n$. But this proves that $f_{1}(y)$ is lower semi-continuous on $A$.

In particular, if $E_{2}=0$, we have that $f(y), y \in A$, is lower semicontinuous on $A$. Thus, if $A=Y$, this shows that $f(y)$ is lower semicontinuous on $Y$.

LEMMA 2. Let $A$ be a connected subspace of $Y$. Let $g_{i}(y), y \in A$, be a (finite) integral-valued, lower semi-continuous function, $i=1, \cdots, n$. Let $g(y)=\sum g_{i}(y), y \in A$, be upper semi-continuous. Then $g_{i}(y)$ is constant on $A$ for each $i$.

Proof. The function $g^{*}(y)=\sum_{j \neq i} g_{j}(y)$ is lower semi-continuous. 
Hence $g_{i}(y)$ is upper semi-continuous, being the difference of an upper semi-continuous function $g(y)$ and a lower semi-continuous function $g^{*}(y)$. Since $g_{i}(y)$ is also lower semi-continuous, it is continuous. Since $A$ is connected, $g_{i}(y)$ is constant on $A$.

Theorem 2. Let $T, A, E(A)$, and $f(y)$ be given as in $\$ 2$. Let $A$ be connected, and suppose that $f(y)$ is upper semi-continuous and $f(y) \leqq k$ $<+\infty, y \in A$. Then $E(A)$ has at most $k$ components. Let these components be $E_{1}, \cdots, E_{r}, r \leqq k$. Let $f_{i}(y), y \in A$, be the number of $\gamma \in S(y)$ such that $\gamma \subset E_{i}$. Then $f_{i}(y)$ is constant for each $i$.

Proof. If $E(A)=0$, the theorem is trivial. If $E(A) \neq 0$, let $E_{1}, \cdots$, $E_{r}$ be such that $E_{i} \neq 0, E_{i} \cdot C\left(E_{j}\right)=0$ if $i \neq j$, and $\sum E_{i}=E(A)$. Let $f_{i}(y), y \in A$, be defined as in the theorem. Clearly $f_{i}(y)$ is integralvalued and $\neq \equiv 0$. Moreover, by Lemma $1, f_{i}(y)$ is lower semi-continuous on $A$. Since $f(y)=\sum f_{i}(y)$ is upper semi-continuous and bounded on $A$, Lemma 2 is applicable, and $f_{i}(y) \equiv k_{i} \geqq 1, i=1, \cdots, r$. But since

$$
k \geqq f(y)=\sum_{i=1}^{r} f_{i}(y) \geqq \sum_{i=1}^{r} 1=r,
$$

we have $r \leqq k$. But if $E(A)$ had more than $k$ components, then there would exist $E_{1}, \cdots, E_{k+1}$ such that $E_{i} \neq 0, E_{i} \cdot C\left(E_{j}\right)=0$ if $i \neq j$, and $\sum E_{i}=E(A)$. Hence $E(A)$ has at most $k$ components and the theorem is true.

\section{Open connected sets $A$.}

THEOREM 3. Let $T: y=t(x), x \in X$, be a continuous transformation from a connected, locally connected, locally compact, separable metric space $X$ into a topological space $Y$. Let $A$ be a connected open set in $Y$, let $E(A)$ be given as in $\$ 2$, and suppose that $f(y) \not \equiv 0$ is upper semicontinuous and bounded on $A$. Let $y_{1} \in A, y_{2} \in A$. Then there exist points $x_{1} \in E\left(y_{1}\right), x_{2} \in E\left(y_{2}\right)$, and an arc $C$ from $x_{1}$ to $x_{2}$ such that $T(C) \subset A$.

Proof. Let $E_{1}(A)$ be any component of $E(A)$. Let $f_{1}(y), y \in A$, be the number of $\gamma \in S(y)$ such that $\gamma \subset E_{1}(A)$. Then by Theorem 2, $f_{1}(y) \equiv k_{1}>0, y \in A$. Hence there exist an $x_{1} \in E\left(y_{1}\right) \cdot E_{1}(A)$ and an $x_{2} \in E\left(y_{2}\right) \cdot E_{1}(A)$. Let $G$ be the component of $T^{-1}(A)$ which contains $E_{1}(A)$. Then $[2$, Theorem $\mathrm{I}, 14.1] G$ is open and connected. Hence [2, Theorems I, 14.3 and II, 5.2] $G$ is arcwise connected. Therefore there exists an arc $C \subset G$ joining $x_{1}$ and $x_{2}$. Clearly $T(C) \subset A$.

6. Plane transformations. Let $T: z=t(w), w \in D$, be a bounded continuous transformation from a bounded connected open set $D$ in 
the w-plane into the z-plane. Radó and Reichelderfer [3, p. 263] have defined essential maximal model continua (e.m.m.c.) $\gamma$ for such transformations. Let $S(z)$ be the class of (disjoint) e.m.m.c. of $z$. If $\gamma \in S(z)$ then $\gamma \subset T^{-1}(z)$ and $S(z)$ has property $\mathrm{P}$ [3, Lemmas 2.8 and 2.16]. Define $E(A)$ by equations (1) and (2). The function $f(z)$ becomes the essential multiplicity function $\kappa(z)$ [3, p. 263]. The conditions in Theorems 1, 2, and 3 are satisfied, and we have the following result.

CoROLlaRY. Let $T: z=t(w)$, weD, be a bounded continuous transformation from a bounded connected open set $D$ in the w-plane into the $z$-plane. Let $\kappa(z)$ be upper semi-continuous on $A$ with $\kappa(z) \leqq k<+\infty$, $z \in A$. Then if $A$ is closed, $E(A)$ is closed; if $A$ is connected, $E(A)$ has at most $k$ components.

Theorem 3 of course yields a corollary also.

Reichelderfer ${ }^{3}$ [4] has recently defined a class $S^{+}(z)$ for such plane transformations as follows. $S^{+}\left(z_{0}\right)$ consists of all e.m.m.c. $\gamma_{0}$ of $z_{0}$ such that if $G$ is any open set containing $\gamma_{0}$ then there exists a finitelyconnected Jordan region $R \subset G$ containing $\gamma_{0}$ in its interior and such that $\mu\left(z_{0}, T, R\right)>0$ (see [3, p. 263] for notation). Reichelderfer [4] shows that $S^{+}(z)$ has property P. Define $E^{+}(A)$ by equations (1) and (2). The function $f(z)$ is then denoted by $\kappa^{+}(z)$. Then Theorems 1,2 , and 3 apply in this case.

Similar definitions and conclusions hold for $E^{-}(A)$ (see [4]).

7. Examples. The discussion will be restricted to bounded continuous plane transformations $T: z=t(w), w \in D$, where $D$ is an open simply-connected set, and to the functions $\kappa(z)$ and $E(z)$ mentioned in $\S 6$. The following examples shed further light on Theorems 1, 2, and 3 . We have let $v=u+i v, z=x+i y$, and have used whichever notation is more convenient.

I. In Theorem 1 , if $f(z)$ is merely assumed to be bounded, then $E(A)$ is not necessarily closed. For let $D$ be the disc $|w|<2$, and let

$$
z=\left\{\begin{array}{lr}
w, & |w| \leqq 1 \\
w /|w|, & 1<|w|<2 .
\end{array}\right.
$$

Let $A$ be the closed disc $|z| \leqq 1$. Then $\kappa(z) \leqq 1, z \in A$, and $E(A)$ is the set $|w|<1$ which is not closed in $D$.

II. In Theorem 1 , if $k(z) \equiv+\infty, z \in A$, then $E(A)$ is not necessarily closed. Sierpinski [5] has constructed a continuous function $x=g^{*}(u)$, $0 \leqq u \leqq 1$, such that $\kappa\left(x, g^{*}\right)=+\infty$ for $0<x<1$ except at a countable

${ }^{3}$ The author had the privilege of reading this paper in manuscript. 
set of points. A slight modification of his function yields a continuous function $x=g(u), 0 \leqq u \leqq 1$, such that $\kappa(x, g) \equiv+\infty, 0<x<1$, and such that $g(1)=1$. To construct the required example, let $D$ be the open rectangle: $0<u<3,0<v<1$. Let $T$ be defined by the relations

$$
\begin{aligned}
& x=\left\{\begin{array}{l}
g(u), \\
(3-u) / 2, \\
1 / 2,
\end{array}\right. \\
& 0<u \leqq 1 \text {, } \\
& y=v, \\
& 1<u \leqq 2 \text {, } \\
& 2<u<3 \text {, } \\
& 0<v<1 \text {. }
\end{aligned}
$$

Let $A$ be the line segment: $1 / 2 \leqq x \leqq 3 / 4, y=1 / 2$. Then $\kappa(z) \equiv+\infty$, $z \in A$, and $E(A)$ is not closed because it contains the segment $3 / 2<u$ $<2, v=1 / 2$, but not the point $u=2, v=1 / 2$.

III. In Theorem 1 , if $A$ is open and $\kappa(z) \equiv k<+\infty, z \in A$, it does not follow that $E(A)$ is open. For let $D$ be the disc $|w|<2$, and let

$$
z=t(w)= \begin{cases}(w-1) w, & 1 \leqq|w|<2, \\ |w|-1, & 0 \leqq|w|<1 .\end{cases}
$$

Let $A$ be the $\operatorname{disc}|z|<2$. Then it follows that $\kappa(z) \equiv 1, z \in A$, and $E(A)$ is the set of points $w$ such that $1 \leqq|w|<2$. Hence $E(A)$ is not open.

IV. In Theorem 2, $E(A)$ may be connected, thus having only 1 component, even though $\kappa(z) \equiv k<+\infty, z \in A$. For let $z=w^{k},|w|<2$. Let $A$ be the unit circle $|z|=1$. Then $\kappa(z) \equiv k, z \in A$. But $E(A)$ is the unit circle $|w|=1$, and is therefore connected.

V. Theorem 3 does not hold for line segments $A$. For let $D$ be the open rectangle: $-2<u<2,-2<v<2$. Let $S$ be the point set consisting of the line segment $u=0,-1 \leqq v \leqq 1$, and the curve $v=\sin 2 \pi / u$, $0<u \leqq 1$. Let $S^{\prime}$ be the point set consisting of $S$ and the two line segments $-2<u<0, v=0$, and $1<u<2, v=0$. Let $d\left((u, v), S^{\prime}\right)$ be the distance from the point $(u, v)$ to the set $S^{\prime}$. Define the transformation $T$ as follows:

$$
\begin{aligned}
& x=\left\{\begin{array}{lr}
u, & -2<u<0, \\
0, & 0 \leqq u \leqq 1, \\
u-1, & 1<u<2,
\end{array}\right. \\
& y=\left\{\begin{array}{lr}
d\left((u, v), S^{\prime}\right), & (u, v) \text { above } S^{\prime}, \\
0, & (u, v) \in S^{\prime}, \\
-d\left((u, v), S^{\prime}\right) & (u, v) \text { below } S^{\prime} .
\end{array}\right.
\end{aligned}
$$

$T$ is clearly continuous. Let $A$ be the line segment: $-1 \leqq x \leqq 1 / 2$, $y=0$. Then $\kappa(z) \equiv 1, z \in A$, and $T^{-1}(A)=E(A)$ consists of the line 
segments $-1 \leqq u<0, v=0$, and $1<u \leqq 3 / 2, v=0$, and the set $S$. Hence if $z_{1}=(-1,0)$ and $z_{2}=(1 / 2,0)$ there is no arc $w_{1} w_{2} \subset D$ such that $t\left(w_{1}\right)=z_{1}, t\left(w_{2}\right)=z_{2}, T\left(\operatorname{arc} w_{1} w_{2}\right) \subset A$.

VI. There remains the question as to whether, in the case of plane transformations, we could require $C \subset E(A)$ in Theorem 3 . This would be impossible in the general case, as may be shown by an example similar to the one described in V.

\section{BIBLIOGRAPHY}

1. S. Lefschetz, Algebraic topology, Amer. Math. Soc. Colloquium Publications, vol. $27,1942$.

2. G. T. Whyburn, Analytic topology, Amer. Math. Soc. Colloquium Publications, vol. $28,1942$.

3. T. Radó and P. V. Reichelderfer, A theory of absolutely continuous transformations in the plane, Trans. Amer. Math. Soc. vol. 49 (1941) pp. 258-307.

4. P. V. Reichelderfer, Law of transformation for essential generalized jacobians, Duke Math. J. vol. 16 (1949) pp. 73-83.

5. W. Sierpinski, Sur une fonction continue dans un intervalle qui prend chaque valeur de cet intervalle $\aleph_{0}$ fois, Publications Mathématiques Univerzitet Belgrade vols. 6 and 7 (1938) pp. 84-85

Ohio State University 